\title{
ABL-N may induce apoptosis of human prostate cancer cells through suppression of KLF5, ICAM-1 and Stat5b, and upregulation of Bax/Bcl-2 ratio: An in vitro and in vivo study
}

\author{
YANPING ZHANG, KAILONG LIU, YONG ZHANG, JINCHUN QI, BAOSAI LU, \\ CHONGJUN SHI, YUEWEI YIN, WENQING CAI and WEI LI \\ Department of Urology, The Second Hospital of Hebei Medical University, Shijiazhuang 050000, P.R. China
}

Received June 10, 2015; Accepted July 17, 2015

DOI: $10.3892 /$ or.2015.4293

\begin{abstract}
Identification of novel botanicals that can selectively induce apoptosis and arrest growth of cancer cells without producing cytotoxic effects is highly appreciable for cancer therapy. The present study aimed to investigate the possibility of acetylbritannilactone (ABL) derivative 5-(5-(ethylperoxy) pentan-2-yl)-6-methyl-3-methylene-2-oxo-2,3,3a,4,7,7a-hexahydroben-zofuran-4-yl2-(6-methoxynaphthalen-2-yl) propanoate $(\mathrm{ABL}-\mathrm{N})$ as a therapeutic agent in human prostate cancer and potential mechanisms. Human prostate cancer cells were treated with ABL-N of different concentrations $(0,5,10,20$, 30 and $40 \mu \mathrm{mol} / \mathrm{l})$. Cell viability, migration and apoptosis were determined. Activities of caspases were assayed, as well as protein expression of cancer-related proteins KLF5, Stat5b and ICAM-1 in PC3 cells. The therapeutic effect of ABL-N was further evaluated in our tumor xenografts. ABL-N inhibited growth of prostate cancer cells in a dose-dependent manner, without obvious effect on normal human prostate epithelial PrEC cells. ABL-N administration induced apoptosis of PC 3 cells in a dose-dependent manner, along with the enhanced activity of caspases and increased $\mathrm{Bax} / \mathrm{Bcl}-2$ ratio. Expression of KLF5, Stat5b and ICAM-1 was significantly downregulated in PC3 cells. Our in vivo study further confirmed that ABL-N significantly inhibited the tumor growth of PC3 cells in the xenograft mouse model. ABL-N induces apoptosis of prostate cancer cells through activation of caspases, increasing the ratio of $\mathrm{Bax} / \mathrm{Bcl}-2$, as well as suppression of KLF5, Stat5b and ICAM-1 expressions. The present study indicated that ABL-N may be a potential therapeutic drug for human prostate cancer, and our data supported further studies to explore the therapeutic potential of ABL-N in other types of human cancer.
\end{abstract}

Correspondence to: Professor Wei Li, Department of Urology, The Second Hospital of Hebei Medical University, 215 Heping West Road, Shijiazhuang 050000, P.R. China

E-mail:weili356@163.com

Key words: ABL-N, prostate cancer, apoptosis, Krüppel-like factor 5, intercellular adhesion molecule 1, Stat $5 \mathrm{~b}, \mathrm{Bax} / \mathrm{Bcl}-2$ ratio

\section{Introduction}

Prostate cancer is the most common malignancy and the second leading cause of cancer-related deaths in many Western countries. It accounts for 27\%/233,000 of incident cases in the US alone with a total of 29,480 predicted deaths, according to cancer statistics reported by the American Cancer Society in 2014 (1). The incidence of prostate cancer in Asian population, including the Chinese, has been increasing in recent years, although still lower than that of Western countries $(2,3)$. The incidence of prostate cancer is known to increase with advancing age, and it inevitably becomes an increasingly greater problem as life expectancy is globally improving.

Apoptosis is an important process in a wide variety of biological systems, including cell development and maintenance of tissue homeostasis, and is well documented to play an essential role as a protective mechanism against carcinogenesis (4). In prostate cancer, a fine balance between cell proliferation and apoptosis is lost contributing to the increased cellular mass and tumor progression (5). Intervention using cancer chemopreventive compounds has shown a promising opportunity for preventing or slowing the progression of this downloaded disease (6). Increasing attention has been focused on the utilization of naturally occurring botanicals or dietary substances for prostate cancer therapy (7-9). In this regard, for prostate cancer chemoprevention at the present time, there is considerable emphasis in identifying novel botanicals that selectively induce apoptosis and growth arrest of prostate cancer cells without producing cytotoxic effects on normal cells.

Acetylbritannilactone (ABL) is a sesquiterpene lactone abundant in Inula Britannica L, a traditional Chinese medicinal herb (Xuan Fu Hua). It has been reported to have chemopreventive properties by inducing cell apoptosis in breast and ovarian cancers $(10,11)$. We have recently synthesized a derivative compound of ABL, 5-(5-(ethylperoxy) pentan-2-yl)-6-methyl-3-methylene-2-oxo-2,3,3a,4,7,7a-hexah ydrobenzofuran-4-yl2-(6-methoxynaphthalen-2-yl) propanoate (ABL-N; Fig. 1A). Our previous study indicated that ABL-N treatment causes a significant inhibition of tumor growth in vivo and ABL-N induces apoptosis in breast cancer cells through the activation of caspases and JNK signaling pathways, 
suggested that ABL-N may be a potential drug for breast cancer prevention and intervention (12). However, the antitumor activity and the molecular targets of $\mathrm{ABL}$ in prostate cancer cells have not been determined. In the present study, we investigated the antiproliferative and pro-apoptotic effects of ABL-N, as well as the expression of apoptosis-related proteins in ABL-induced growth suppression of human prostate cancer cells and the xenograft mouse model.

\section{Materials and methods}

Cell lines. The human prostate cancer cells PC3, DU145 and LNcap, were purchased from the American Type Culture Collection (ATCC; Manassas, VA, USA) and were routinely cultured in RPMI-1640 supplemented with $10 \%$ fetal bovine serum (FBS) (both from Invitrogen, Carlsbad, CA, USA), $4 \mathrm{mmol} / \mathrm{l}$ glutamine, $100 \mathrm{U} / \mathrm{ml}$ penicillin and $100 \mu \mathrm{g} / \mathrm{ml}$ streptomycin. The normal epithelial prostate cells PrEC were obtained from Clonetics-BioWhittaker, Inc. (Walkersville, MD, USA), were cultured in prostate epithelial basal medium with PrEGM BulletKit (both from Clonetics). All cells were incubated at $37^{\circ} \mathrm{C}$ and $5 \% \mathrm{CO}_{2}$ in a humid environment and subcultured twice a week.

$A B L-N$ preparation. ABL-N was prepared as described in our previous study (12). The purity and chemical structure of ABL-N were certified by melting point, elemental analysis and spectral studies. The purified ABL-N was dissolved in ethanol using ultrasonication. The effects of ABL-N on our experiments were compared with those of ethanol at a final concentration of $0.5 \%$ as vehicle.

MTT assay. The PC3, DU145, LNcap and PrEC cells were seeded into 96 -well plates at a density of 4,000/well and were cultured for $24 \mathrm{~h}$. Cells were then treated with ABL-N of different concentrations $(0,2.5,5,10,15,20,25,30,35$ and $40 \mu \mathrm{M})$. After $24 \mathrm{~h}$ of incubation, the 3-(4,5-dimethylthiazol2-yl)-2,5-diphenyltetrazolium bromide (MTT) reagent, $5 \mathrm{mg} / \mathrm{ml}$ in phosphate-buffered saline (PBS) was added to each well ( $20 \mu \mathrm{l} /$ well) followed by incubation for $4 \mathrm{~h}$ at $37^{\circ} \mathrm{C}$, and then the plate was centrifuged at $1,000 \mathrm{rpm}$ for $5 \mathrm{~min}$ at $4^{\circ} \mathrm{C}$. After careful removal of the medium, formazan crystals were dissolved in $0.1 \mathrm{ml}$ buffered dimethylsulfoxide (DMSO; Sigma), and the absorbance was read on a microplate reader at the wavelength of $570 \mathrm{~nm}$. Absorbance values were normalized to the values obtained from the vehicle-treated cells. Taking into account the more aggressive and highly metastatic nature of prostate cancer, PC3 cells were selected as a model system to conduct further experiments.

Wound migration assay. Confluent PC 3 cells grown in $10 \mathrm{~cm}^{2}$ dishes were wounded using sterile pipette tips, washed twice with 1X PBS, and grown in RPMI-1640 medium (Invitrogen) supplemented with $10 \%$ FBS and various concentrations of ABL-N for $24 \mathrm{~h}$. Then PC3 cells were photographed under a phase-contrast microscope (magnification, $\mathrm{x} 10$ ).

Apoptosis assays. Apoptosis of PC3 cells was firstly determined using terminal deoxynucleotidyl transferasemediated nick-end labeling (TUNEL) kit (DeadEnd
Fluorometric TUNEL System; Promega, Madison, WI, USA) after cultivation with different concentrations of ABL-N for $24 \mathrm{~h}$. Cells were fixed in $4 \%$ paraformaldehyde in PBS for $25 \mathrm{~min}$ at $4^{\circ} \mathrm{C}$. After permeabilized in $0.1 \%$ Triton $\mathrm{X}-100$ in PBS for $5 \mathrm{~min}$ on ice, the samples were incubated in TUNEL reaction mixture for $1 \mathrm{~h}$ at $37^{\circ} \mathrm{C}$ in a dark and humidified atmosphere.

For nuclear staining, the fixed cells were placed on slides and stained with $1 \mathrm{mg} / \mathrm{ml}$ DAPI for $15 \mathrm{~min}$. After three washes, images were captured immediately using a digital camera attached to the fluorescence microscope.

For quantification of apoptosis by flow cytometry, PC3 cells were grown at a density of $70-80 \%$ confluency and treated with different concentrations of ABL-N for $24 \mathrm{~h}$. The cells were trypsinized, washed with PBS and were processed for labeling with Annexin $\mathrm{V}$ and propidium iodide (PI) using an Annexin V-FLUOS staining kit (Roche Diagnostic Corporation) according to the manufacturer's protocol. The labeled cells were analyzed by flow cytometry (Becton-Dickinson, San Jose, CA, USA).

Caspase activity assay. The activities of caspase-2, -3, -6 and -8 were separately assayed using the respective Caspase-Glo assays (Promega) according to the manufacturer's protocol. Briefly, cells were solubilized with lysis buffer for fluorometric assay. After incubation at $37^{\circ} \mathrm{C}$ for $1 \mathrm{~h}$, the caspase-2, $-3,-6$ and -8 activities were monitored by measuring the fluorescence at $460 \mathrm{~nm}$. Each sample was measured in triplicates.

Western blot analysis. PC3 cells were treated with ABL-N, harvested and lysed. Equal amounts of cell extracts were separated using SDS-PAGE and transferred to a PVDF membrane. The protein was visualized using the enhanced chemiluminescence kit (Amersham Biosciences) as previously described (13).

Tumor xenograft experiments. The 4-week-old athymic male nude mice $(\mathrm{BALB} / \mathrm{c})$ were purchased from the Vital River Laboratory Animal Technology Co., Ltd. [certificate no. SCXK (Jing) 2007-0001]. An aliquot of $1 \times 10^{6}$ PC3 cells suspended in $50 \%$ Matrigel were implanted subcutaneously into both flanks of each BALB/c mouse. Six days after tumor cell inoculation, small tumors were identified. Animals were randomly divided into experiment and control groups $(\mathrm{n}=6), \mathrm{ABL}-\mathrm{N}(15 \mathrm{mg} / \mathrm{kg}$ body weight) or an equal volume of the vehicle was intraperitoneally injected, respectively. Tumor growth was assessed every other day by caliper measurement and tumor volume was estimated by the formula width ${ }^{2} \mathrm{x}$ length $\mathrm{x} 1 / 2$. At the time of sacrifice, tumors were excised and a portion fixed in $10 \%$ buffered formalin for $24 \mathrm{~h}$ for immunohistochemical studies. The animal study was approved by the Ethics Committee for animal research of Hebei Medical University. All the animals were bred and maintained in the Specific Pathogen Free Animal Care Facility. The National Institutes of Health guidelines for the care and use of laboratory animals were followed in all animal procedures.

Immunohistochemistry. Portions of the dissected mouse tumors were immediately fixed in $10 \%$ neutral buffered formalin for $24 \mathrm{~h}$ at room temperature after harvesting, and 


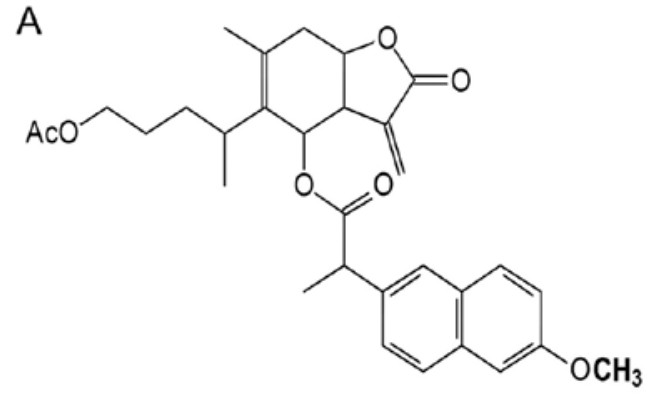

C

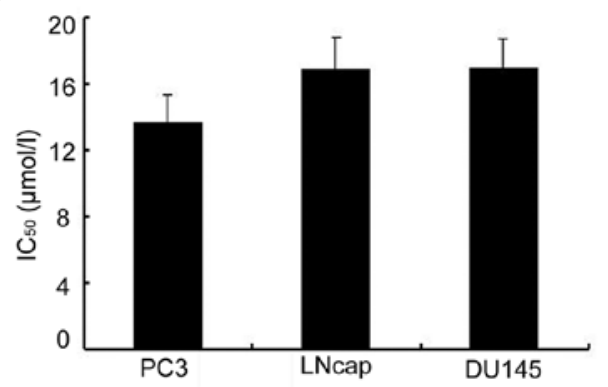

$\mathrm{D}$
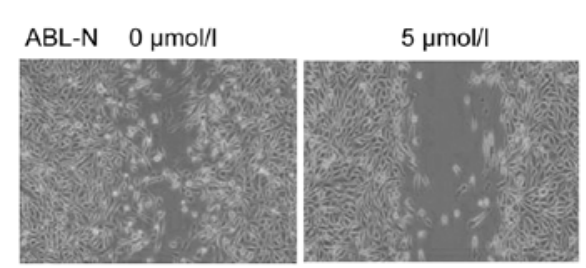

B

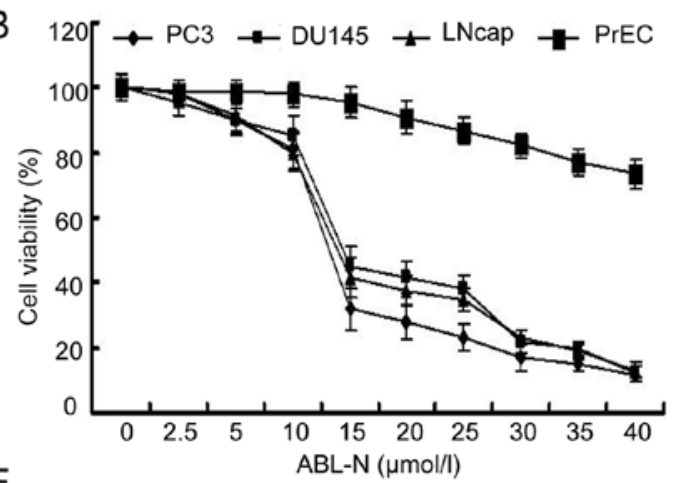

E

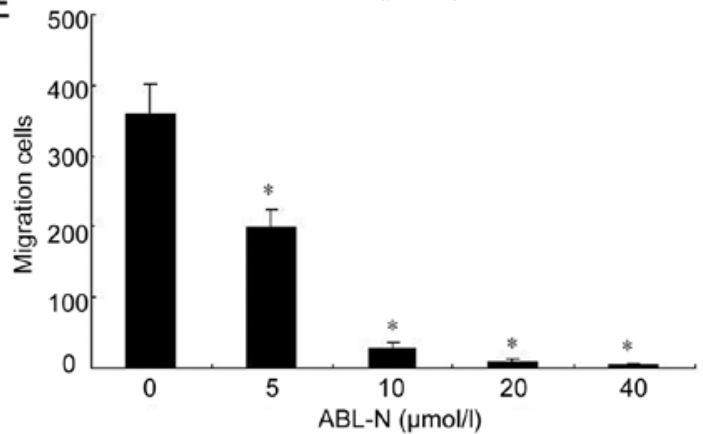

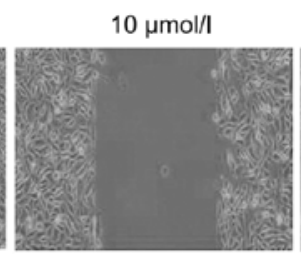
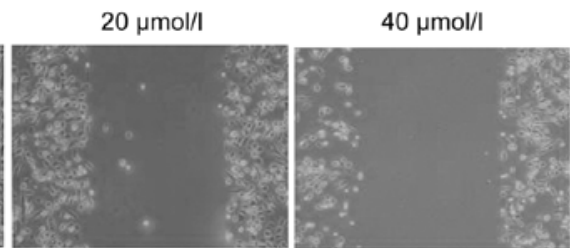

Figure 1. ABL-N and its effect on cell viability and migration of human prostate cell lines. (A) The chemical structures of ABL-N; (B) human prostate cancer cells PC3, DU145, LNcap and normal human prostate epithelial cells PrEC were treated with various concentrations of ABL-N for 24 h and cell viability was determined by MTT assay. The values were represented as the percent viable cells, with vehicle-treated cells regarded as $100 \%$ viable. $(\mathrm{C}) \mathrm{IC}_{50}$ values of cancer cells PC3, LNcap and DU145 for ABL-N. The $\mathrm{IC}_{50}$ value is the concentration of ABL-N that reduces the cell viability by $50 \%$ under the experimental conditions. (D) Migration assay of PC3 cells treated with different concentrations of ABL-N. Cells in three representative fields of panel were counted, and data from three independent experiments were summarized as histograms. (E) PC3 cells were grown to confluency. The cell layer was scraped and photographed after cultured in various concentration of ABL-N for $24 \mathrm{~h}$. Photomicrographs of representative 20 fields. Results represent the means \pm SE from three independent experiments. ${ }^{*} \mathrm{p}<0.05$ vs. the vehicle-treated ones.

were then placed in $70 \%$ ethanol. Formalin-fixed tissues were embedded in paraffin, sectioned at $5 \mu \mathrm{m}$ and incubated with the specific antibodies against Stat5b, KLF5, ICAM-1, Bcl-2 and Bax (Santa Cruz Biotechnology) for $1 \mathrm{~h}$ at room temperature, followed by biotinylated secondary antibodies for $30 \mathrm{~min}$ at room temperature. Sections were counterstained with hematoxylin. The specimens were viewed with an Olympus BX51 microscope. Staining intensities were determined by measurement of the integrated optical density (IOD) with light microscopy using a computer-based Image-Pro Morphometric system. Measurements were conducted by two independent observers in a double-blind manner.

Statistical analysis. Data are expressed as the means \pm SE. ANOVA and the paired or unpaired t-test was performed for statistical analysis as appropriate. $\mathrm{p}<0.05$ was considered to indicate a statistically significant result.

\section{Results}

$A B L-N$ inhibits the cell viability of PC3, DU145 and LNCap cells. MTT assay showed that ABL-N suppressed the cell viability of human prostate cancer cells (PC3, DU145 and LNcap) in a dose-dependent manner (Fig. 1B), with similar $\mathrm{IC}_{50}$ values obtained $24 \mathrm{~h}$ after ABL-N treatment (Fig. 1C). However, the survival of normal human prostate epithelial PrEC cells was minimally affected by ABL-N treatment, even at high concentrations $(40 \mu \mathrm{mol} / \mathrm{l})$ that were highly cytotoxic to the prostate cancer cells (Fig. 1B).

ABL-N inhibits the cell migration of PC3 cells. According to the experimental results of MTT, the final concentrations of $0,5,10,20$ and $40 \mu \mathrm{mol} / 1$ were used for ABL-N treatment in the further experiments. In the wound-healing assay, ABL-N treatment significantly decreased wound healing of PC3 cells in a dose-dependent manner when compared with the control cells (Fig. 1D and E).

ABL-N induces apoptosis of PC3 cells. To investigate whether ABL-N reduced cell viability involving the induction of cell apoptosis, we conducted flow cytometric analysis and TUNEL assay of ABL-N-treated PC3 cells. As shown in Fig. 2, in the cells treated with ABL-N, the percentage of cells stained positive for Annexin $\mathrm{V}$ and negative for PI 
A

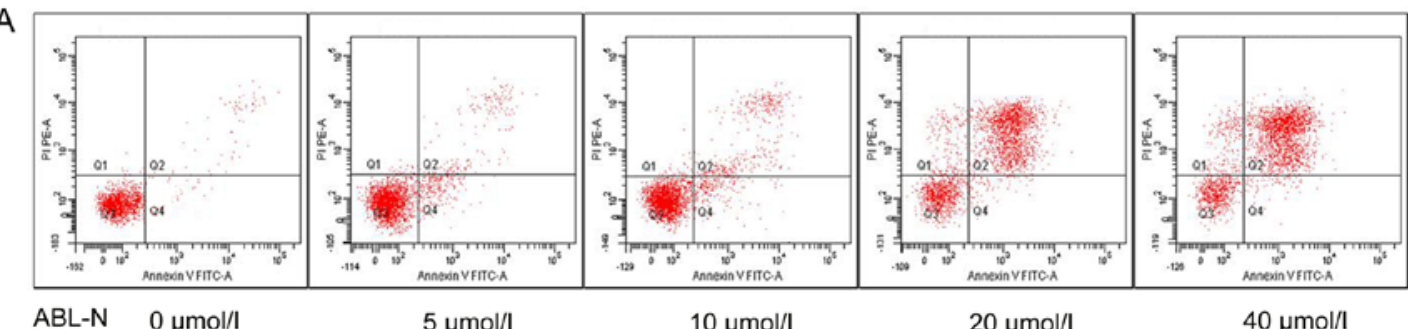

B

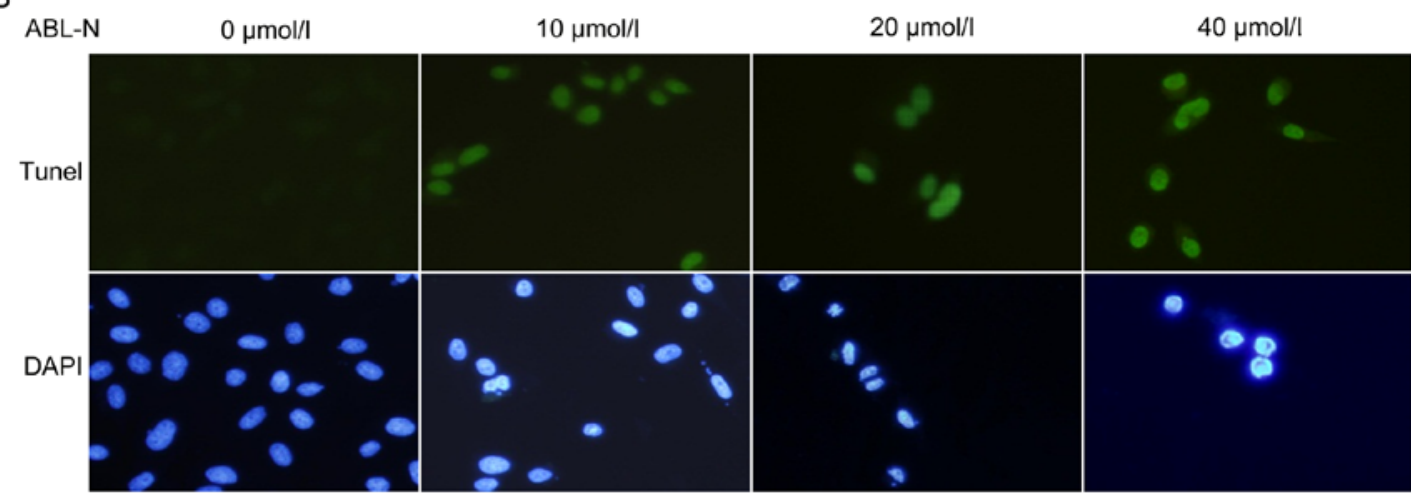

C

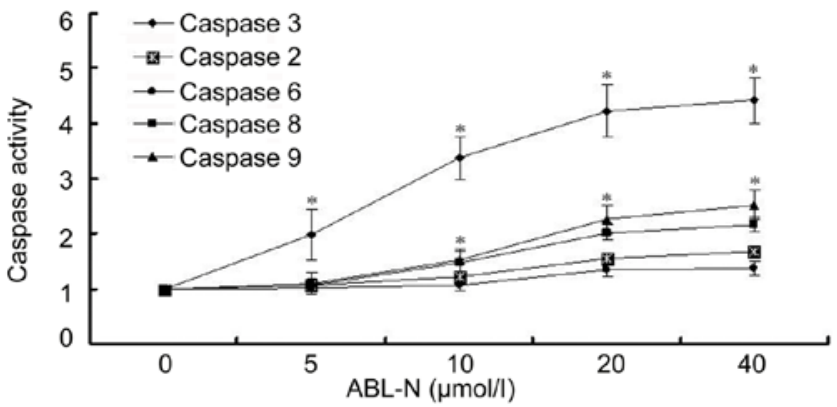

Figure 2. Effect of ABL-N on cell apoptosis in prostate cancer cells. PC3 cells were harvested after treated with different concentrations of ABL-N (0, 5 , 10, 20 and $40 \mu \mathrm{mol} / \mathrm{l}$ ) for $24 \mathrm{~h}$. (A) Apoptosis of PC3 cells treated with ABL-N as determined by Annexin V/PI staining. Percentages of negative (viable), Annexin V-positive (early apoptotic), PI-positive (necrotic) or Annexin V and PI double-positive (late apoptotic) cells, are shown by a flow cytometric analysis (B) Representative micrographs of PC3 cells undergoing apoptosis were stained for TUNEL to assess the effects of ABL-N on apoptosis. Nuclear condensation is shown by DAPI-staining assay. (C) The activation of caspases was detected. The data are expressed as the means \pm SE of three separate experiments. ${ }^{*} \mathrm{p}<0.05$ vs. the vehicle-treated ones.

was significantly increased after ABL-N treatment, even at the lower concentrations. The number of Annexin V and PI-positive cells was significantly increased at higher concentrations of ABL-N (20 and $40 \mu \mathrm{mol} / 1$, Fig. 2A). Cells were further stained for TUNEL and DAPI to assess the effects of ABL-N on apoptosis, results showed enhanced apoptosis of PC3 cells after exposure to ABL-N, and the condensed and fragmented nuclei increased with ABL-N treatment (Fig. 2B).

$A B L-N$ induces the activities of caspases in PC 3 cells. To test whether caspases were involved in the ABL-N-induced apoptosis, the activities of caspase- $2,-3,-6$ and -8 were colorimetrically assayed. As shown in Fig. 2C, caspase-3 activity was significantly increased after ABL-N treatment and was dose-dependent. The activities of caspase- 8 and -9 were also enhanced to some extent, while this effect was less significant than that of the caspase- 3 . By contrast, caspase- 2 and -6 activities were not significantly influenced by ABL-N treatment, even at the high concentration of $40 \mu \mathrm{mol} / 1$ (Fig. 2C).
ABL-N inhibits cancer-related proteins in PC3 cells. Western blotting was performed to investigate the expression of cancer-related proteins Stat5b and Klf5 and pro-angiogenic factor ICAM-1 in ABL-N-treated PC3 cells. As shown in Fig. 3A, ABL-N treatment significantly decreased the expression of Stat5b, KLF5 and ICAM-1, and this effect was enhanced with the increasing concentration of ABL-N $(\mathrm{p}<0.05)$.

$A B L-N$ treatment results in an elevated $B a x / B c l-2$ ratio in $P C 3$ cells. The ratio of Bax/Bcl2 is often considered as a decisive factor in cell apoptosis or survival. As shown in Fig. 3B, the exposure of human PC 3 cells to ABL-N caused a marked increase in Bax protein expression, while the levels of Bcl-2 protein were not obviously affected after ABL-N treatment. This resulted in a substantial increase in $\mathrm{Bax} / \mathrm{Bcl}-2$ ratio, which favors apoptosis and collectively forms a molecular basis for the apoptotic action of ABL-N (Fig. 3B).

$A B L-N$ inhibits the growth of prostate cancer in vivo. The xenograft models of PC3 cells were established in 

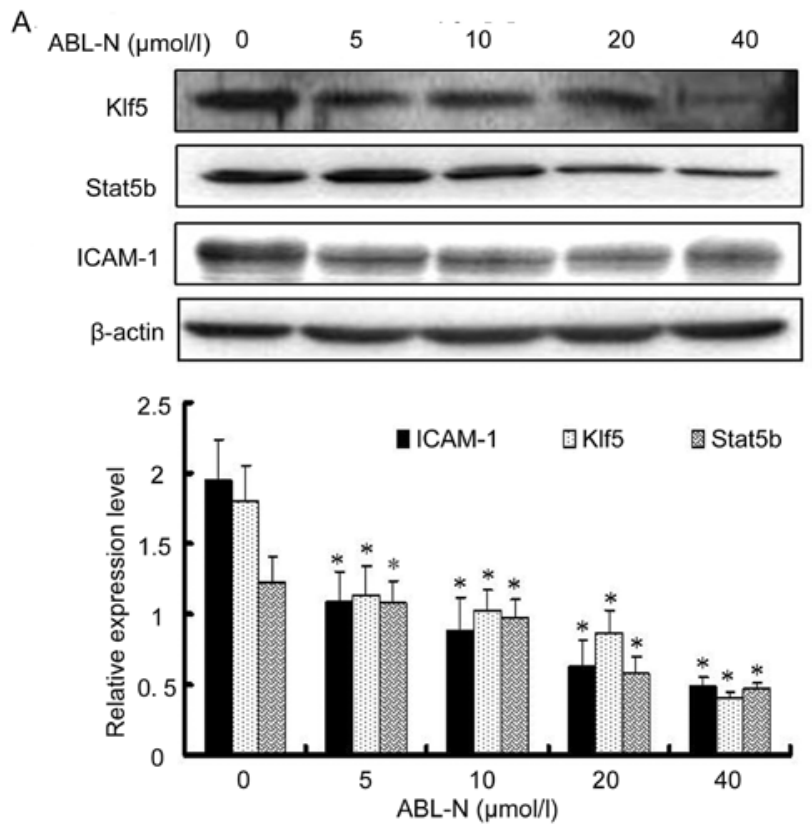

B
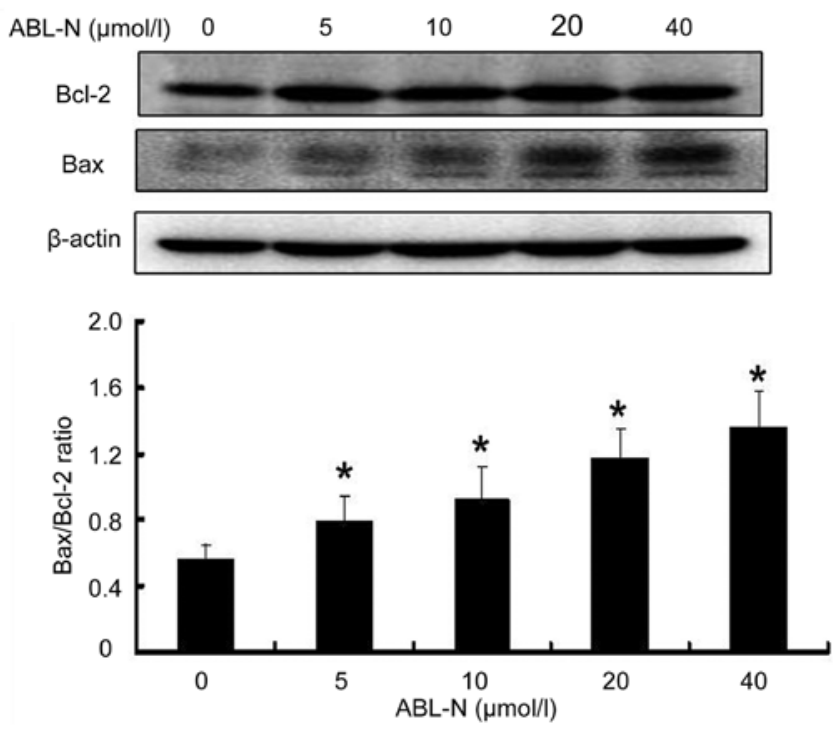

Figure 3. Effects of ABL-N on the expression of cancer-related proteins and $\mathrm{Bax} / \mathrm{Bcl}-2$. PC3 cells were treated with vehicle alone or specified concentrations of ABL-N $(0,5,10,20$ and $40 \mu \mathrm{mol} / 1)$ for $24 \mathrm{~h}$. (A) Protein levels of cancer-related proteins KLF5, ICAM-1 and Stat5b in PC3 cells as detected by western blotting. Equal loading of protein was confirmed by stripping and reprobing the blots with $\beta$-actin. Data from three independent experiments are summarized as a histogram. (B) Effect of ABL-N on Bax/Bcl-2 ratio in PC3 cells. The data obtained from the western blot analysis were used to evaluate the effect of $\mathrm{ABL}-\mathrm{N}$ on the $\mathrm{Bax} / \mathrm{Bcl}-2$ ratio. The densitometric analysis of Bax and Bcl-2 bands was performed using TotalLab TL120 software, and the data (relative density normalized to $\beta$-actin) were plotted as Bax/Bcl-2 ratio. Data are expressed as the means $\pm \mathrm{SE}$ of three separate experiments with similar results. ${ }^{*} \mathrm{p}<0.05$ vs. the vehicle-treated ones.

BALB/c mice to investigate the effect of ABL-N on tumor growth in vivo. As shown in Fig. 4, treatment with ABL-N $(15 \mathrm{mg} / \mathrm{kg})$ significantly suppressed the growth of tumor cells when compared with the vehicle-treated control group $(\mathrm{p}<0.05)$. No toxicity was observed and treated mice showed no weight loss, decreased activity or anorexia (data not shown). These data indicated beneficial therapeutic effect of ABL-N in the xenograft prostate cancer mouse model.

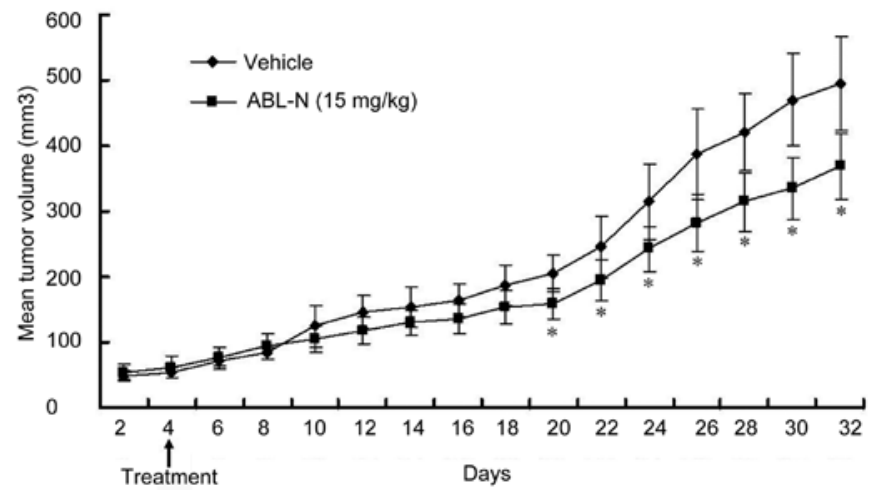

Figure 4. Effect of ABL-N on the growth of PC3 xenografts. PC3 nude mouse xenograft model was used with ABL-N $(15 \mathrm{mg} / \mathrm{kg})$ or vehicle $(10 \%$ DMSO in water), and tumors volumes were measured with calipers on alternate days. Points, mean tumor volume in each experimental group containing 6 mice; bars, SD; ${ }^{*}$ p $<0.05$ vs. the vehicle-treated ones. DMSO, dimethylsulfoxide.

ABL-N modulates Bcl-2/Bax and inhibits KLF5, Stat5 and ICAM-1 in the PC3 xenograft mouse model. ABL-N treatment was found to inhibit or decrease the tumorigenic potential of PC3 cells in vivo, and we further determined the effect of ABL-N administration on the expression levels of Bax and Bcl-2, as well as KLF5, Stat5b and ICAM-1 in tumors excised from PC3 xenograft mice. In accordance with the results obtained in vitro, immunohistochemical analysis showed that Bax expression was significantly enhanced in tumor tissues of animals treated with $\mathrm{ABL}-\mathrm{N}$, and the level of $\mathrm{Bcl}-2$ protein was not significantly altered after ABL-N treatment $(\mathrm{p}<0.01$, Fig. 5A and B). Moreover, tumor sections from ABL-Nadministered mice exhibited significantly decreased protein expression of KLF5, Stat5b and ICAM-1 when compared with vehicle-treated ones ( $p<0.05$, Fig. 5C and D).

\section{Discussion}

Recently, the apoptosis signaling systems have been shown to provide promising targets for development of novel anticancer agents (14). Several plant-derived bioactive agents, such as delphinidin, baicalein, gambogic acid and green tea, are known as chemopreventive agents and have been reported to induce apoptosis in a number of experimental models of carcinogenesis (15). Induction of apoptosis is therefore considered as a possible therapeutic mechanism of these chemopreventive agents. ABL, which has been shown to be potently antitumorigenic, has pro-apoptotic features in numerous carcinoma cell types $(11,16)$. We recently obtained a highly active derivative ABL-N, which has shown exceptional antiproliferative activity in human breast cancer cells (12). The aim of the present study was to investigate the possible role of ABL-N-induced apoptosis in human prostate cancer cells and delineate the potential mechanism.

Our results showed that ABL-N inhibited the cell viability of human prostate cancer cell lines LNCaP, DU145 and PC3 at low concentration, and after increased treatment with $20 \mu \mathrm{M}$ or more of ABL-N, there was a pronounced accumulation of apoptotic cells. By contrast, cell viability of normal human prostate epithelial PrEC cells was not significantly influenced by ABL-N even at high concentration, indicating that ABL-N 
A

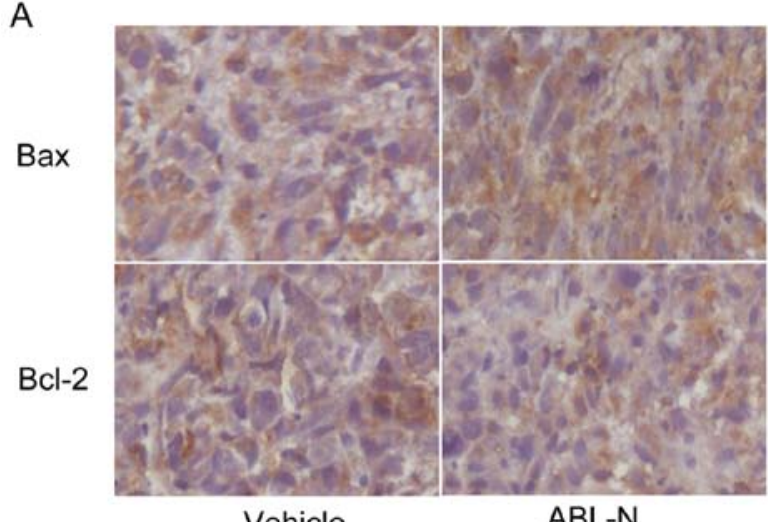

Vehicle

C

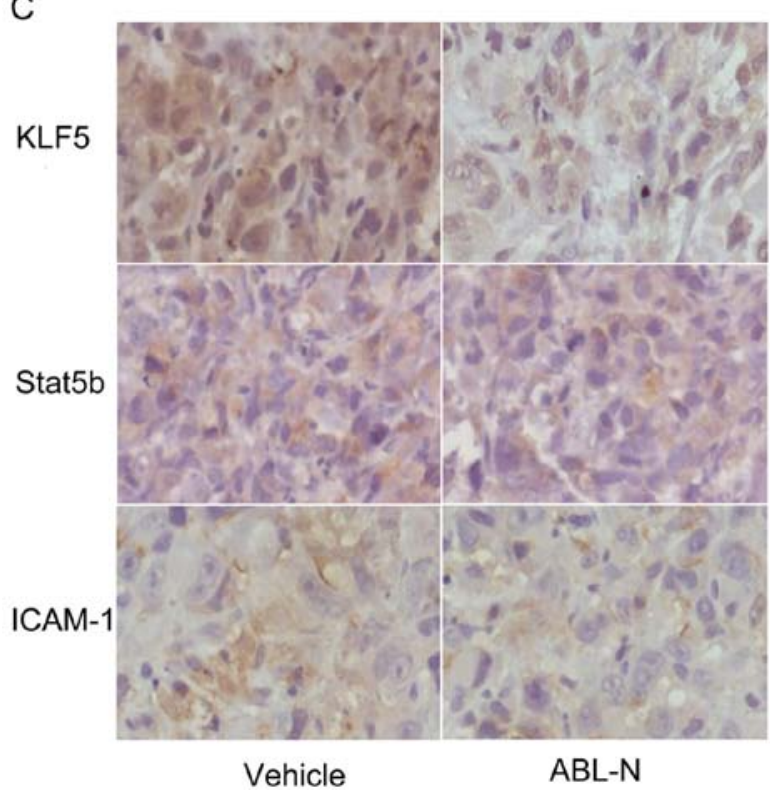

B

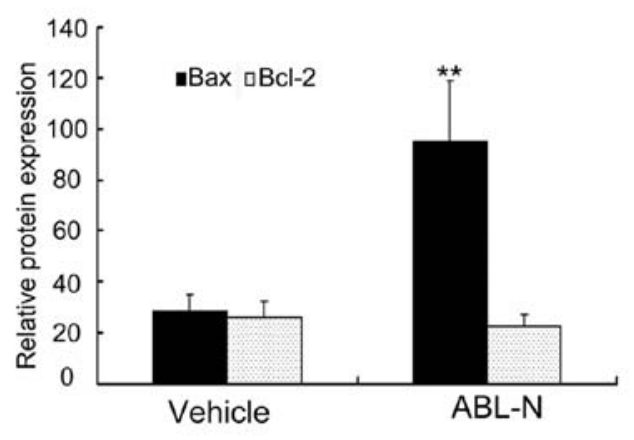

D

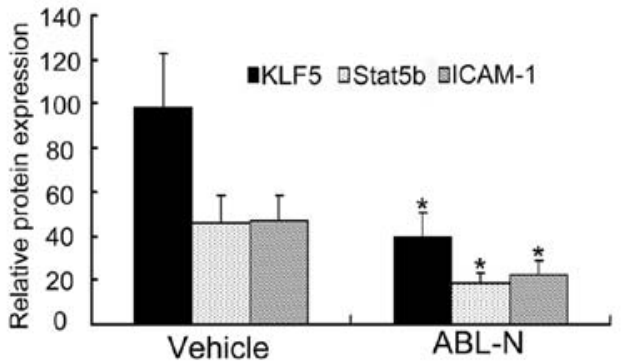

Figure 5. Effect of ABL-N administration on the expression of Bcl-2 and Bax, as well as cancer-related protein KLF5, Stat5 and ICAM-1 in PC3 xenograft mice. Immunohistochemical analysis of protein expressions of (A) Bax and Bcl-2, as well as (B) Stat5b, ICAM-1 and KLF5 in tumor sections of vehicle- or ABL-N-treated mice. Staining intensities were determined by measurement of the integrated optical density (IOD) with light microscopy using a computer-based Image-Pro Morphometric system (C and D, respectively). ${ }^{*} \mathrm{p}<0.05$ and ${ }^{* *} \mathrm{p}<0.01$ vs. the vehicle-treated ones. (A and B) Magnification, $\mathrm{x} 200$.

selectively induced apoptosis and arrested growth of prostate cancer cells without producing cytotoxic effects on normal cells. Studies in our laboratories have previously reported the death of different human breast cancer cell lines induced by ABL-N, and this effect has been linked to caspase-dependent apoptosis (12). Results of our TUNEL and DAPI assays demonstrated that ABL-N induced apoptosis in PC3 cells. The number of Annexin V and PI-positive cells increased with higher concentrations of ABL-N, indicating an activation of the pro-apoptotic pathway with consecutive apoptotic cell death. In the quantification of apoptosis by flow cytometry experiments, the number of the cells actively undergoing apoptosis during ABL-N exposure was determined to investigate the sensitivity of prostate cancer cells to ABL-N-induced apoptosis. After exposure to $40 \mu \mathrm{mol} / 1 \mathrm{ABL}-\mathrm{N}$, however, the late apoptotic/necrotic cells predominated with high proportion of $65 \%$ compared with that of $15 \%$ for early apoptotic cells, indicating the accelerated cell death induced by ABL-N treatment.

Caspases are known as key mediators of apoptosis and contributed to the apoptotic morphology through the cleavage of various cellular substrates. Caspase-3 is an executioner caspase that can be activated by a mitochondrial pathway involving activation of caspase-9 due to release of cytochrome $c$ to the cytosol or a death receptor pathway involving caspase- 8 (17). In the present study, the results that caspase- 3 activation enhanced markedly indicated that caspase-3 plays a key role as an important executioner in ABL-N-induced apoptosis in PC3 cell lines. Moreover, the results of the present study indicated that ABL-N-induced apoptosis in PC-3 cells is probably mediated by both caspase- 9 and caspase- 8 . Consequently, we hypothesized that mitochondria or death receptor-mediated activation of the caspases may be a potential mechanism underlying ABL-N-induced apoptosis in prostate cancer cells and further study was performed.

Bcl-2 family has been shown to play an important regulatory role in apoptosis, either as activator (Bax) or as inhibitor (Bcl-2) $(14,18)$. Bax exerts pro-apoptotic activity by translocation from the cytosol to the mitochondria, where it induces cytochrome $c$ release, while $\mathrm{Bcl}-2$ exerts its anti-apoptotic activity, at least in part by inhibiting the translocation of Bax to the mitochondria $(19,20)$. The Bcl-2 and Bax 
protein ratio has been recognized as a key factor in regulation of the apoptotic process $(14,21)$. They can activate or inhibit the release of downstream factors such as cytochrome $c$ which leads to the activation of caspase-3 and PARP in the execution of apoptosis (22). The results of the present study indicated that ABL-N-induced apoptosis in human PC3 cells was accompanied by upregulation of Bax, yet with no marked downregulation effect on Bcl-2 protein expression.

Signal transducer and activator of transcription $5 \mathrm{a}$ and $5 \mathrm{~b}$ (Stat5a/b) is critical for the viability of human prostate cancer cells, and it is activated in prostate cancer, yet not in normal human prostate epithelium (23). The activation of Stat5a/b in primary prostate cancer predicted early prostate cancer recurrence (24). It has been reported that active Stat 5 promoted migration and invasion of prostate cancer cells, induced re-arrangement of the microtubule network and increased metastases formation of prostate cancer cells $(25,26)$. Pro-angiogenic factor ICAM-1 has been reported to provide a structural and functional interface between epithelial cells and the extracellular environment, and the expression of ICAM-1 in PC3 cells was correlated with increased metastatic potential of prostate cancer cells (27). Herein, we also showed that expression of ICAM-1 and Stat5b was significantly decreased in highly metastatic PC-3 prostate cancer cells treated with ABL-N.

KLF5 is a basic transcriptional factor that functions in multiple cellular processes including cell proliferation, differentiation and apoptosis, and it has both pro- and antitumorigenic effects (28). KLF5 is thought to be a tumor suppressor in prostate and breast cancers $(29,30)$, while it has also been shown to drive proliferation in cultured cells and to be a prognostic factor for the survival of patients with breast cancer (31). Studies that examine KLF5 expression by stage reflected high KLF5 expression in early stages of cancer progression and lower KLF5 expression in later stages $(32,33)$. Our results showed that ABL-N apparently decreased the protein expression of KLF5 in a dose-dependent manner. Thus, KLF5 also plays a promoting role in the process of prostate cancer progression. Accordingly, we hypothesized that ABL-N antitumor effect is possibly through inhibiting these factors and further study was needed to demonstrate this effect.

We next used an experimental model of prostate cancer to further evaluate the antitumor effect of ABL-N and the potential mechanism. The findings of our in vivo study confirmed the antitumor activity of ABL-N against the PC3 human xenografts, a widely accepted model of highly aggressive prostate cancer. Administration of ABL-N for only 12 consecutive days inhibited the growth of established PC3 tumors when compared with that of the vehicle-treated ones. ABL-N treatment did not result in significant changes in body weights and histologic data in nude mice. Corroborating the results in vitro, our in vivo study also demonstrated the upregulated expression of Bax and increased Bax to Bcl-2 ratio, as well as the decreased expression of cancer-related proteins KLF5, Stat5b and pro-angiogenic factor ICAM-1. The present study provided clear experimental evidence that ABL-N exerted therapeutic and preventive effects on prostate cancer without notable toxicity, and it acts through suppression of KLF5, ICAM-1 and Stat5b expression and upregulation of Bax/
$\mathrm{Bcl}-2$ ratio. All the above results indicated that ABL-N may be a promising candidate for cancer therapy, although further experimental studies should be performed to demonstrate the therapeutic potential of ABL-N in other types of cancer and the functional mechanism.

To the best of our knowledge, this is the first study detailing apoptosis induction of ABL-N in prostate cancer cell $\mathrm{PC} 3$ in vivo and in vitro. Our results indicated that $\mathrm{ABL}-\mathrm{N}$ inhibited proliferation of PC-3 cells at least partly by causing apoptosis through suppressing the cancer-related protein Stat5b, Klf5 and ICAM-1, and increasing Bax/Bcl-2 ratio indicating that ABL-N may be developed as a potential anticancer agent against human prostate cancer. The present study offers new therapeutic perspective to prostate cancer therapy, and our data also support further studies to explore the therapeutic potential of ABL-N in other types of human cancer.

\section{Acknowledgements}

The authors express their thanks to Professor Jinkun Wen and Professor Mei Han in the Institute of Basic Medicine, Hebei Medical University for their assistance in the present study.

\section{References}

1. Siegel R, Ma J, Zou Z and Jemal A: Cancer statistics, 2014. CA Cancer J Clin 64: 9-29, 2014.

2. Matsuda T and Saika K: Comparison of time trends in prostate cancer incidence (1973-2002) in Asia, from cancer incidence in five continents, Vols IV-IX. Jpn J Clin Oncol 39: 468-469, 2009.

3. Peng P, Gong Y, Bao P, Ke JZ, Xiang YM, Zhang ML and Zheng Y: Estimates and prediction of prostate cancer incidence, mortality and prevalence in China, 2008. Zhonghua Liu Xing Bing Xue Za Zhi 33: 1056-1059, 2012 (In Chinese).

4. Yin PH, Liu X, Qiu YY, Cai JF, Qin JM, Zhu HR and Li Q: Antitumor activity and apoptosis-regulation mechanisms of bufalin in various cancers: New hope for cancer patients. Asian Pac J Cancer Prev 13: 5339-5343, 2012.

5. Bilbro J, Mart M and Kyprianou N: Therapeutic value of quinazoline-based compounds in prostate cancer. Anticancer Res 33: 4695-4700, 2013.

6. Schmitz-Dräger BJ, Lümmen G, Bismarck E and Fischer C: Prevention strategies for prostate cancer. Minerva Urol Nefrol 64: 225-231, 2012.

7. Samarghandian S, Samini F and Taghavi M: Antiproliferative and cytotoxic properties of honey in human prostate cancer cell line (PC-3): Possible mechanism of cell growth inhibition and apoptosis induction. Afr J Pharm Pharmacol 8: 9-15, 2014.

8. Hafeez BB, Siddiqui IA, Asim M, Malik A, Afaq F, Adhami VM, Saleem M, Din M and Mukhtar H: A dietary anthocyanidin delphinidin induces apoptosis of human prostate cancer PC3 cells in vitro and in vivo: Involvement of nuclear factor-kappaB signaling. Cancer Res 68: 8564-8572, 2008.

9. Yun JM, Kweon MH, Kwon H, Hwang JK and Mukhtar H: Induction of apoptosis and cell cycle arrest by a chalcone panduratin A isolated from Kaempferia pandurata in androgenindependent human prostate cancer cells PC3 and DU145. Carcinogenesis 27: 1454-1464, 2006.

10. Fang XM, Liu B, Liu YB, Wang JJ, Wen JK, Li BH and Han M: Acetylbritannilactone suppresses growth via upregulation of krüppel-like transcription factor 4 expression in HT-29 colorectal cancer cells. Oncol Rep 26: 1181-1187, 2011.

11. Rafi MM, Bai NS, Chi-Tang-Ho, Rosen RT, White E, Perez D and Dipaola RS: A sesquiterpenelactone from Inula britannica induces anti-tumor effects dependent on Bcl-2 phosphorylation. Anticancer Res 25: 313-318, 2005.

12. Liu B, Han M, Sun RH, Wang JJ, Zhang YP, Zhang DQ and Wen JK: ABL-N-induced apoptosis in human breast cancer cells is partially mediated by c-Jun $\mathrm{NH}_{2}$-terminal kinase activation. Breast Cancer Res 12: R9, 2010. 
13. Dong L-H, Wen J-K, Liu G, McNutt MA, Miao SB, Gao R, Zheng B, Zhang H and Han M: Blockade of the Ras-extracellular signal-regulated kinase $1 / 2$ pathway is involved in smooth muscle $22 \alpha$-mediated suppression of vascular smooth muscle cell proliferation and neointima hyperplasia. Arterioscler Thromb Vasc Biol 30: 683-691, 2010.

14. Rao L and White E: Bcl-2 and the ICE family of apoptotic regulators: Making a connection. Curr Opin Genet Dev 7: 52-58, 1997.

15. Yi T, Yi Z, Cho SG, Luo J, Pandey MK, Aggarwal BB and Liu M: Gambogic acid inhibits angiogenesis and prostate tumor growth by suppressing vascular endothelial growth factor receptor 2 signaling. Cancer Res 68: 1843-1850, 2008

16. Bai N, Lai CS, He K, Zhou Z, Zhang L, Quan Z, Zhu N, Zheng QY, Pan MH and Ho CT: Sesquiterpene lactones from Inula britannica and their cytotoxic and apoptotic effects on human cancer cell lines. J Nat Prod 69: 531-535, 2006.

17. Wolf BB and Green DR: Suicidal tendencies: Apoptotic cell death by caspase family proteinases. J Biol Chem 274: 20049-20052, 1999.

18. $\mathrm{Hu} \mathrm{W}$ and Kavanagh JJ: Anticancer therapy targeting the apoptotic pathway. Lancet Oncol 4: 721-729, 2003.

19. Wang X: The expanding role of mitochondria in apoptosis. Genes Dev 15: 2922-2933, 2001

20. Wolter KG, Hsu YT, Smith CL, Nechushtan A, Xi XG and Youle RJ: Movement of Bax from the cytosol to mitochondria during apoptosis. J Cell Biol 139: 1281-1292, 1997.

21. Adams JM and Cory S: The Bcl-2 protein family: Arbiters of cell survival. Science 281: 1322-1326, 1998.

22. Tafani M, Schneider TG, Pastorino JG and Farber JL: Cytochrome $c$-dependent activation of caspase- 3 by tumor necrosis factor requires induction of the mitochondrial permeability transition. Am J Pathol 156: 2111-2121, 2000.

23. Ahonen TJ, Xie J, LeBaron MJ, Zhu J, Nurmi M, Alanen K, Rui $\mathrm{H}$ and Nevalainen MT: Inhibition of transcription factor Stat5 induces cell death of human prostate cancer cells. J Biol Chem 278: 27287-27292, 2003.

24. Li H, Zhang Y, Glass A, Zellweger T, Gehan E, Bubendorf L, Gelmann EP and Nevalainen MT: Activation of signal transducer and activator of transcription-5 in prostate cancer predicts early recurrence. Clin Cancer Res 11: 5863-5868, 2005.
25. Gu L, Vogiatzi P, Puhr M, Dagvadorj A, Lutz J, Ryder A, Addya S, Fortina P, Cooper C, Leiby B, et al: Stat5 promotes metastatic behavior of human prostate cancer cells in vitro and in vivo. Endocr Relat Cancer 17: 481-493, 2010

26. Gu L, Dagvadorj A, Lutz J, Leiby B, Bonuccelli G, Lisanti MP, Addya S, Fortina P, Dasgupta A, Hyslop T, et al: Transcription factor Stat3 stimulates metastatic behavior of human prostate cancer cells in vivo, whereas Stat5b has a preferential role in the promotion of prostate cancer cell viability and tumor growth. Am J Pathol 176: 1959-1972, 2010.

27. Yoo NC, Chung HC, Chung HC, Park JO, Rha SY, Kim JH, Roh JK, Min JS, Kim BS and Noh SH: Synchronous elevation of soluble intercellular adhesion molecule-1 (ICAM-1) and vascular cell adhesion molecule-1 (VCAM-1) correlates with gastric cancer progression. Yonsei Med J 39: 27-36, 1998.

28. Li X, Zhang B, Wu Q, Ci X, Zhao R, Zhang Z, Xia S, Su D, Chen J, Ma G, et al: Interruption of KLF5 acetylation converts its function from tumor suppressor to tumor promoter in prostate cancer cells. Int J Cancer 136: 536-546, 2015.

29. Chen C, Bhalala HV, Vessella RL and Dong JT: KLF5 is frequently deleted and down-regulated but rarely mutated in prostate cancer. Prostate 55: 81-88, 2003.

30. Chen C, Sun X, Ran Q, Wilkinson KD, Murphy TJ, Simons JW and Dong JT: Ubiquitin-proteasome degradation of KLF5 transcription factor in cancer and untransformed epithelial cells Oncogene 24: 3319-3327, 2005.

31. Tong D, Czerwenka K, Heinze G, Ryffel M, Schuster E, Witt A, Leodolter $\mathrm{S}$ and Zeillinger R: Expression of $K L F 5$ is a prognostic factor for disease-free survival and overall survival in patients with breast cancer. Clin Cancer Res 12: 2442-2448, 2006.

32. Kwak MK, Lee H-J, Hur K, Park J, Lee HS, Kim WH, Lee KU, Choe KJ, Guilford P and Yang HK: Expression of Krüppel-like factor 5 in human gastric carcinomas. J Cancer Res Clin Oncol 134: 163-167, 2008.

33. McConnell BB, Bialkowska AB, Nandan MO, Ghaleb AM, Gordon FJ and Yang VW: Haploinsufficiency of Krüppel-like factor 5 rescues the tumor-initiating effect of the $A p c^{M i n}$ mutation in the intestine. Cancer Res 69: 4125-4133, 2009. 